\title{
REGULARIZACIÓN FORMAL DEL GÉNERO EN LOS ADJETIVOS DEL CATALÁN EN COMPARACIÓN CON EL ESPAÑOL*
}

\author{
Jesús Jiménez \\ Universitat de València
}

\author{
Maria-Rosa Lloret \\ Universitat de Barcelona
}

\section{RESUMEN}

Con la desaparición del género neutro en el paso del latín a las lenguas románicas, los adjetivos latinos de tres terminaciones se adaptaron en catalán como adjetivos de dos terminaciones, normalmente con las marcas $\varnothing$ para el masculino y - $a$ para el femenino, como en clar-Ø 'claro' - clar-a 'clara'; este patrón es transparente y máximamente icónico. Los adjetivos de dos terminaciones, en cambio, se convirtieron en invariables, como en catalán antiguo fort- $\varnothing$ 'fuerte'. El sincretismo de género, a pesar de los problemas de iconicidad y transparencia que genera, podría mantenerse si las clases invariables estuvieran definidas por propiedades extramorfológicas que contribuyeran a su estabilidad, como sucede en espańol con la terminación - $e$, que permite adscribir los adjetivos a la clase de invariables (por ej., fuerte). En catalán, sin embargo, la ausencia de terminaciones específicas para los invariables agrava los problemas de iconicidad y transparencia de estas formas, por lo que la tendencia a crear femeninos analógicos (como fort-a 'fuerte, FEM.') es más fuerte que en español.

Palabras Clave: analogía, flexión, género, morfología natural, sincretismo, catalán, español.

\section{GENDER FORMAL REGULARIZATION IN THE ADJECTIVES \\ OF CATALAN IN COMPARISON WITH SPANISH}

\section{Abstract}

Due to the loss of the neutral gender in the evolution from Latin to Romance languages, three-ending Latin adjectives were adapted as two-ending adjectives in Catalan, usually with the marks $\varnothing$ in the masculine and $-a$ in the feminine, as in clar 'clear, MAsc.' - clar- $a$ 'clear, FEM.'; this pattern is transparent and maximally iconic. Two-ending Latin adjectives became invariable instead, as in old Catalan fort- $\varnothing$ 'strong', giving rise to gender syncretic forms, which lack transparency and iconicity. Despite the naturalness challenges raised by syncretism, it can be maintained if invariable classes are defined by extramorphological properties that enhance their stability, as is the case of Spanish with the ending - $e$ determining membership to the invariable gender class (e.g., fuert-e 'strong'). In Catalan, though, the lack of exclusive endings for invariable adjectives worsens their iconicity and transparency, and so the tendency to create analogical feminine forms (e.g., fort-a 'strong, FEM.') is stronger than in Spanish.

KEYWORDs: analogy, gender, inflection, natural morphology, syncretism, Catalan, Spanish.

DOI: https://doi.org/10.25145/j.refiull.2020.40.06

Revista de Filología, 40; enero 2020, pp. 105-124; ISSN: e-2530-8548 


\section{INTRODUCCIÓN}

El catalán, al igual que otras lenguas romances como el español, presenta dos clases de adjetivos en relación con el género (sobre esta cuestión, v., por ejemplo, Fabra 1956: \$\$28-30; Mascaró 1985: 85-102; Clua 2002: 485-507; AVL 2006: 115-119; Lloret 2013; IEC 2016: 169-175)1. Por una parte, encontramos adjetivos de dos terminaciones, variables respecto del género, con distinción formal entre el género masculino y el femenino (1). En los adjetivos variables de dos terminaciones la forma femenina acaba siempre en $-a$, una implicación ya apuntada por Fabra (1956: 20). Por lo que respecta al masculino, la clase más numerosa, con diferencia, es la que no tiene ninguna marca explícita, esto es, presenta el morfo $\varnothing$ para el masculino, como en (1a). También existen masculinos acabados en - $e$ (1b) $y$, aun menos frecuentemente, en $-o$ (1c) y en $-u$ (realizada como [w]) (1d). Igual que sucede con los adjetivos variables terminados en $-a$, las formas acabadas en - $o$ y en $-u(1 d)$ corresponden unívocamente a adjetivos masculinos. Por otra parte, encontramos adjetivos de una terminación, invariables en cuanto al género, con una única forma para el masculino y para el femenino. En este caso, la clase más frecuente es la de los adjetivos sin marca explícita de flexión, cuya forma se reduce al radical (2a) (IEC 2016: 171); también existe un grupo relativamente importante de adjetivos invariables acabados en $-e(2 \mathrm{~b})$ y un pequeño grupo de adjetivos acabados en $-a(2 c)^{2}$.

$\begin{array}{ll}\text { a. clar- } \varnothing & \text { 'claro' } \\ \text { àrid- } \varnothing & \text { 'árido' } \\ \text { ferm- } \varnothing & \text { 'firme, MASc.' } \\ \text { fort- } \varnothing & \text { 'fuerte, MASc.' } \\ \text { verd- } \varnothing & \text { 'verde, MASc.' } \\ \text { cortés- } \varnothing & \text { 'cortés, MASC.' }\end{array}$

clar-a
àrid-a
ferm-a
fort-a
verd-a
cortes-a

\begin{abstract}
'clara'
'árida'

'firme, FEM.'

'fuerte, FEM.'

'verde, FEM.'

'cortés, FEM.'
\end{abstract}

* Esta investigación forma parte del proyecto FFI2016-76245-C3-3-P, financiado por la Agencia Estatal de Investigación y por el FEDER (http://www.ub.edu/GEVAD); del grupo de investigación consolidado 2017SGR942, financiado por la Generalitat de Catalunya, y del grupo GIUV2013137, de la Universitat de València. Agradecemos los comentarios de los asistentes al XV Encuentro de Morfólogos Espańoles (Universidad de La Laguna, 9-10/05/2019), así como las sugerencias de dos revisores anónimos y de Elena Felíu y Manuel Pérez Saldanya.

${ }^{1}$ Por simplicidad expositiva, para poder comparar con mayor facilidad los ejemplos del catalán con los del español, a lo largo del trabajo tomamos la pronunciación occidental como referencia para las formas del catalán. Por lo que atañe a las clases morfológicas, esta variedad distingue, como el español, las terminaciones $-e$ y $-a$ y las terminaciones $-o$ y $-u$ en posición átona, tal como reflejan las formas ortográficas; en catalán oriental, la diferencia es meramente ortográfica, ya que las terminaciones - $e$ y $-a$ se neutralizan en [ə] y las terminaciones $-o$ y $-u$, en $[\mathrm{u}]$. Además, en el caso de las terminaciones en $-e$, no distinguiremos los casos en que $e$ se puede interpretar como una vocal epentética, como ampl-e o notabl-e ( $c f .{ }^{*}$ ampl, ${ }^{*}$ notabl), de los casos en que la marca -e no se puede justificar por criterios fonológicos, como còmod-e o enorm-e (cf. àrid- $\varnothing$, ferm- $\varnothing$ ).

${ }_{2}$ Existen, como en español, otras clases invariables muy minoritarias que no tratamos en el trabajo, como algunas formas acabadas en vocal tónica ( $h i n d \dot{u}$, papú) o en -i (cursi, ianqui 'yanqui'). 

b. ampl-e
'ancho'
ampl-a
'ancha'
negr-e
'negro'
còmod-e
'cómodo'
negr-a
'negra'
c. fof-o
'fofo'
d. ate-u
'ateo'
còmod-a 'cómoda'
a. escolar- $\varnothing \quad$ 'escolar, MASc. y FEM.'
fof-a
'fofa'
ate-a
'atea'
fàcil- $\varnothing$
'fácil, MASC. y FEM.'
b. notabl-e
simpl-e
'notable, MASC. y FEM.'
'simple, MASC. y FEM.'
enorm-e
'enorme, MASC. y FEM.'
c. belg-a
'belga, MASC. y FEM.'
agrícol-a
'agrícola, MASC. y FEM.'

Respecto del origen de estas formas (sobre esta cuestión, v., entre otros, Badia 1951: \$117; Moll 1952: \$1.3.2; Casanova 1983, 1985), las formas variables suelen derivar de adjetivos latinos de tres terminaciones: con la desaparición del género neutro en el paso del latín a las lenguas romances, estos adjetivos se adaptaron como adjetivos de dos terminaciones en catalán, normalmente con la marca $\varnothing$ para el masculino y la marca - a para el femenino; por ej., clar < CLARUM $~$ clara < CLARAM. Los adjetivos invariables del catalán, en cambio, suelen provenir de los adjetivos de dos terminaciones del latín, que ya presentaban una forma idéntica para el masculino y para el femenino; por ej., escolar < SCHOLAREM, fàcil < FACILEM. Existen algunos adjetivos con variación entre el masculino y el femenino en latín que se han convertido en invariables en catalán, como simple < SIMPLUS, -A, -UM o doble 'doble' < Duplus, -A, -UM (Badia 1951: \$67.II, \$121.II; DECat III: 194, vir: 925926). El patrón inverso, la formación de femeninos analógicos a partir de adjetivos originalmente invariables, es muy frecuente en todos los estadios del catalán, como muestra la creación de los femeninos forta o verda a partir de las formas invariables fort < FORTEM y verd < VIRIDEM. Es decir, el catalán, como otras lenguas romances, tiende a regularizar algunas de las formas sincréticas del modelo de (2) según el modelo con variación de (1).

Teniendo esto en cuenta, nuestro objetivo en este trabajo es describir los problemas estructurales de la falta de distinción formal entre el masculino y el femenino en catalán y compararlos con los problemas que presenta el español (sección 2). Para ello, mostraremos, en primer lugar, los problemas de transparencia y de iconicidad del sincretismo de género y los beneficios para el sistema del modelo con distinción de género y de su extensión analógica a otros adjetivos (sección 2.1). Además, mostraremos que en catalán la ausencia de una marca específica para los adjetivos invariables complica la definición de esta clase formal y favorece la regularización analógica de los adjetivos invariables, mucho más que en español, con un sistema más estable (sección 2.2). La sección 3 se dedica a la presentación de los patrones de regularización típicos del catalán, que incluyen la creación tanto de femeninos como, en menor medida, de masculinos analógicos; estas regularizaciones se ponen en relación con los procesos equivalentes del español. En esta sección, además, demostramos documentalmente que los procesos analógicos aparecen en diferentes etapas de 
la lengua y que el cambio se difunde de manera gradual, palabra a palabra, a través del léxico. El trabajo se cierra con las conclusiones (sección 4).

\section{PROBLEMAS DE NATURALIDAD DE LAS FORMAS SINCRÉTICAS EN CATALÁN}

En este apartado presentamos los problemas de naturalidad de las formas sincréticas de (2) en catalán y los comparamos con los de las formas equivalentes del español. Enmarcamos nuestra discusión dentro de los formalismos de la morfología natural propuestos principalmente por Dressler $(1985,1987)$, Mayerthaler (1987, 1988) y Wurzel $(1987,1989)$, y aplicados a la morfología del catalán en trabajos como Wheeler (1993), Pérez Saldanya (1998) o Pérez Saldanya et al. (2004). Los problemas que presentamos se dividen en dos categorías: problemas independientes del sistema, relacionados sobre todo con la falta de una marca que exprese la mayor especificidad del femenino (sección 2.1), y problemas dependientes del sistema, derivados de las dificultades de asignación de algunas terminaciones a una única clase formal, variable o invariable (sección 2.2).

\subsection{Falta de transparencia y DE iconicidad formal DE LOS ADJETIVOS INVA- RIABLES}

Las clases morfológicas flexivas tienden a seguir los principios de naturalidad dependientes e independientes del sistema (v. Mayerthaler 1987, 1988; Wurzel 1987, 1989; Wheeler 1993: 98; Pérez Saldanya 1998: 24; Pérez Saldanya et al. 2004). En el segundo grupo, el principio de uniformidad favorece los sistemas en los que cada función es expresada por una única forma (en los que no hay sinonimia o alomorfía) y el principio de transparencia requiere que cada forma corresponda a una sola función (que no haya sincretismo) ${ }^{3}$. En el estudio de los adjetivos invariables, nos interesa especialmente la demanda relativa a la transparencia, según la cual las formas flexivas no deben ser ambiguas, es decir, cada forma debe corresponder a un único significado, como en (3a); por lo tanto, como indicamos en (3b), las formas invariables, con una sola forma para el masculino y el femenino, suponen siempre una transgresión de este principio.

(3) Transparencia formal de los adjetivos del catalán

a. Formas transparentes: dos formas, dos géneros:

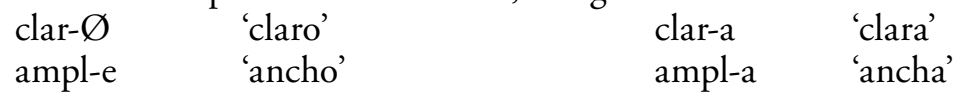

${ }^{3}$ Ambas exigencias se derivan del principio conocido como universal de Humboldt, formulado por Vennemann (1978: 259), que se resume en la máxima «una forma - un significado». 


\begin{tabular}{|c|c|c|}
\hline fof-o & 'fofo' & fof-a \\
\hline ate-u & 'ateo' & ate-a \\
\hline
\end{tabular}

b. Formas no transparentes: una forma, dos géneros:

$\begin{array}{llll}\text { escolar- } \varnothing & \text { 'escolar, MASc.' } & \text { escolar- } \varnothing & \text { 'escolar, FEM.' } \\ \text { notabl-e } & \text { 'notable, MASc.' } & \text { notabl-e } & \text { 'notable, FEM.' } \\ \text { belg-a } & \text { 'belga, MAsc.' } & \text { belg-a } & \text { 'belga, FEM.' }\end{array}$

Además, las clases flexivas tienden a obedecer el llamado principio de iconicidad construccional, que favorece los sistemas en que los elementos no marcados semánticamente -más generales- no tienen marca formal y los elementos marcados semánticamente -más específicos- se señalan con la presencia de una marca formal, o sea, se construyen ańadiendo una marca formal al elemento no marcado. La iconicidad de las construcciones se puede clasificar en una escala de cinco grados de iconicidad (v. Mayenthaler 1988: 18; Wurzel 1989: 11, y la aplicación a la morfología verbal del catalán de Wheeler 1993 y Pérez Saldanya 1998). Como mostramos en (4), las formas de los adjetivos del catalán permiten ilustrar tres de esos cinco grados: el primero, el tercero y el cuarto: ${ }^{4}$

(4) Jerarquía de iconicidad construccional aplicada a los adjetivos del catalán

a. Grado 1, formas con iconicidad máxima (diagramática, con adición segmental): la categoría marcada semánticamente se obtiene añadiendo una desinencia a la no marcada. En el caso del género en catalán, en el patrón mayoritario la forma del masculino, más general, no tiene marca y sirve de base para la formación del femenino, más específico semánticamente y marcado formalmente: clar- $\varnothing$ 'claro'
clar-a
'clara'

b. Grado 3, formas con iconicidad mínima (metafórica, modulatoria): la categoría semánticamente marcada tiene una forma diferente de la no marcada, pero no por la adición de segmentos. En el caso del género de los adjetivos en catalán, en algunos patrones menos comunes que el

${ }^{4}$ Fuera del sistema de adjetivos del catalán, en el grado segundo encontramos formas que no llegan a ser máximamente icónicas (diagramáticas, con adición modular), en que la categoría marcada se forma por la adición de un rasgo prosódico a la no marcada, como en la diferencia del latín entre Domus 'casa' y DOMU:s 'casas', que se basa en la mayor duración de la segunda vocal. En el grado quinto, el peor en cuanto a la iconicidad formal, encontramos formas contraicónicas (sustractivas), en las que la forma semánticamente marcada es segmentalmente más corta que la no marcada; el ejemplo típico que se cita en la bibliografía es la distinción latina entre PHENOMENON 'fenómeno' y phenomena 'fenómenos', en que la forma del singular es más larga que la del plural. En relación con el género, se podrían incluir en este patrón los sustantivos abell-ot- $\varnothing$ 'zángano' o perdig-ot- $\varnothing$ 'perdigón', del catalán, y perdig-ón- $\varnothing$, del español, más largos que sus femeninos respectivos abell-a 'abeja', perdiu- $\varnothing$ 'perdiz' y perdiz, respectivamente. Con todo, estos casos también se podrían incluir en la categoría de formas máximamente icónicas, ya que el femenino es la forma con un significado más genérico y, siguiendo los principios de iconicidad construccional, sirve de base para la formación del masculino, más específico semánticamente y morfológicamente más marcado. 
modelo $\varnothing \sim-a$, la forma del masculino y la del femenino son diferentes, pero ambas tienen una complejidad estructural equivalente, puesto que solo se diferencian por el cambio en la vocal final:

$\begin{array}{llll}\text { ampl-e } & \text { 'ancho' } & \text { ampl-a } & \text { 'ancha' } \\ \text { fof-o } & \text { 'fofo' } & \text { fof-a } & \text { 'fofa' } \\ \text { ate-u } & \text { 'ateo' } & \text { ate-a } & \text { 'atea's }\end{array}$

c. Grado 4, formas no icónicas (sincretismo): la categoría semánticamente marcada no es formalmente diferente de la no marcada. Esto es, ambas formas son iguales:

$\begin{array}{llll}\text { escolar- } \varnothing & \text { 'escolar, MAsc.' } & \text { escolar- } \varnothing & \text { 'escolar, FEM.' } \\ \text { simpl-e } & \text { 'simple, MASC.' } & \text { simpl-e } & \text { 'simple, FEM.' } \\ \text { agrícol-a } & \text { 'agrícola, MASC.' } & \text { agrícol-a } & \text { 'agrícola, FEM.' }\end{array}$

Encontramos, pues, frente a la falta de transparencia y de iconicidad de los adjetivos invariables del catalán, dos modelos variables con mayor iconicidad formal: el modelo mayoritario, máximamente icónico, con alternancia $\varnothing \sim-a$, y un segundo modelo, menos frecuente y mínimamente icónico, con alternancia entre la vocal $a$ del femenino y otra vocal $-e$, $o$ o $u$ - para el masculino.

En relación con la iconicidad de los adjetivos, el español presenta también tres tipos de variantes (5) (sobre esta cuestión, v., por ej., RAE 1973: 191-194; RAE-ASALE 2009: 930-937): formas máximamente icónicas, como cordobés- Ø cordobes-a; mínimamente icónicas, como anch-o - anch-a, y sin iconicidad formal, como cortés- $\varnothing$.

(5) Jerarquía de iconicidad construccional aplicada a los adjetivos del español

a. Grado 1, formas con iconicidad máxima (diagramática, con adición segmental):

$$
\text { cordobés- } \varnothing \quad \text { cordobes-a }
$$

b. Grado 3, formas con iconicidad mínima (metafórica, modulatoria):

$\begin{array}{ll}\text { anch-o } & \text { anch-a } \\ \text { liant-e } & \text { liant-a }\end{array}$

c. Grado 4, formas no icónicas (sincretismo):

$\begin{array}{ll}\text { cortés- } \varnothing & \text { escolar- } \varnothing \\ \text { fuert-e } & \text { belg-a }\end{array}$

Considerando el listado de formas, pues, las dos lenguas son iguales, ya que ambas tienen formas que no son ni icónicas ni transparentes (formas sincréticas). Si solo actuaran los principios independientes del sistema estudiados en este punto,

${ }^{5}$ En ateu - atea, el masculino tiene menos sílabas ([a.téw]) que el femenino ([a.té.a]) y, por lo tanto, se podría considerar como un ejemplo del grado 2, que no llega a ser máximamente icónico porque el femenino se obtiene, además de por la modificación de la vocal, por la adición de una categoría prosódica (v. nota 4). 
ambas lenguas deberían presentar una tendencia similar a convertir en icónicas y transparentes las formas invariables.

\subsection{Estabilidad de LAS Clases EN LOS ADJETivos DEL CATALÁN Y DEL ESPAÑol}

A pesar de su heterogeneidad, sistemas con diferentes patrones como los que hemos descrito en el apartado anterior podrían ser estables si cada clase estuviera definida independientemente por propiedades extramorfológicas, de acuerdo con el principio de estabilidad de las clases definido por Wurzel (1987, 1989); esto es, si los hablantes tuvieran indicios suficientes de algún tipo -formales o semánticos- para asignar cada adjetivo a una clase formal específica. Ahora bien, además de los problemas de transparencia y de iconicidad formal en cuanto al género, los adjetivos del catalán y, en menor medida, del español tampoco están completamente perfilados en clases a partir de una propiedad formal o semántica, como mostramos a continuación.

En español, la correspondencia entre las diferentes terminaciones y una de las clases formales en relación con el género -variable o invariable- es grosso modo bastante precisa, tal como se ilustra en (6).

(6) Implicaciones formales de las terminaciones en el sistema de adjetivos del español

a. Implicación 1: las formas con masculino en -o son variables:

$$
\text { anch-o, anch-a vasc-o, vasc-a }
$$

b. Implicación 2: las formas con masculino en - $a$ son invariables:

c. Implicación 3: las formas acabadas en -e son invariables:

$$
\text { belg-a agrícol-a }
$$

$\begin{array}{ll}\text { fuert-e } & \text { simpl-e } \\ \text { conquens-e } & \text { gigant-e }\end{array}$

Pero, esporádicamente:

$\begin{array}{ll}\text { guap-et-e } & \text { guap-et-a } \\ \text { brut-ot-e } & \text { brut-ot-a } \\ \text { liant-e, } & \text { liant-a }\end{array}$

d. Implicación 4: las formas con masculino acabado en consonante son invariables:

$\begin{array}{ll}\text { feliz- } \varnothing & \text { elemental- } \varnothing \\ \text { fácil- } \varnothing & \text { mejor- } \varnothing \\ \text { azul- } \varnothing & \text { común- } \varnothing \\ \text { cortés- } \varnothing & \text { fiel- } \varnothing\end{array}$

Pero:

saltarín- $\varnothing$, saltarin-a

llorón- $\varnothing$, lloron-a

creador- $\varnothing$, creador-a

receptor- $\varnothing$, receptor-a

tensor- $\varnothing$, tensor-a

cordobés- $\varnothing$, cordobes-a

andaluz- $\varnothing$, andaluz-a

catalán- $\varnothing$, catalan-a

español-Ø, español-a

sajón- $\varnothing$, sajon-a 
Para empezar, los adjetivos con la terminación - $o$ en masculino corresponden sin excepciones a formas variables del modelo $-o--a$ (6a). Según la RAE (1973: 192), "[e]n este paradigma, cada uno de los miembros presupone por regla general al otro, de tal forma que un nombre terminado en - $o$ privado de la forma correlativa en -a es en español casi siempre nombre sustantivo: hominicaco, cuadradillo", y no un adjetivo. Este grupo de mínima iconicidad -o - - $a$ es la clase variable más común en español. Son, por el contrario, invariables en cuanto al género las formas cuyo masculino acaba en $-a$, como belga (6b); se trata de un grupo poco frecuente, pero bien definido formalmente. Los adjetivos acabados en -e, mucho más comunes que los del grupo anterior, son también invariables por regla general (6c). En esta clase encontramos sobre todo formas provenientes de adjetivos de dos terminaciones en latín, como fuerte, pero también algún adjetivo que pertenecía a la clase que distinguía en esa lengua el femenino y el masculino, como simple, firme o libre. Existen algunos ejemplos, escasos, con el patrón de alternancia $-e--a$, que, en conjunto, no sirven para cuestionar la adscripción general de las formas con la terminación -e a la clase de adjetivos invariables. Por una parte, formas con moción de género como guapete o brutote no invalidan la afirmación de que los adjetivos acabados en -e son invariables, porque la marca de género en estos casos se debe a la adición de sufijos expresivos variables, como -ete (guap-et-e, -a; moren-et-e, -a) y -ote (brut-ot-e, -a; tosc-ot-e, $-a$ ). Por otra, formas como liant-e - liant-a, muy minoritarias, tampoco llegan a poner en duda la asociación entre la terminación - e y la clase invariable.

Finalmente, las formas acabadas en consonante en el masculino, que provienen sobre todo de adjetivos de dos terminaciones en latín, son las que plantean más dificultades para su adscripción a una clase formal específica en español (6d). En su mayor parte corresponden a adjetivos invariables: encontramos en este grupo adjetivos acabados en -az, -iz, -oz, como feliz; en los sufijos - al y -ar, como elemental; adjetivos llanos acabados en -il, como fácil; comparativos como mejor, y varios adjetivos aislados como azul, común, cortés o fiel. Existen, sin embargo, bastantes excepciones como los adjetivos acabados en los sufijos expresivos -in (saltarín, -a) o -ón (llorón, -a); en las terminaciones -dor (creador, -a), -tor (receptor, -a) o -sor (tensor, $-a$ ), y los gentilicios formados con el sufijo -és (cordobés, -a), además de otros gentilicios terminados también en consonante como andaluz, - $a$; catalán, -a; espanol, $-a$; sajón, $-a$. Vemos, pues, que, aunque los adjetivos acabados en consonante en el masculino singular pueden ser variables o invariables, existen pistas formales que apuntan en una dirección o en la otra. Por lo tanto, podemos concluir que, en conjunto, la adscripción de los adjetivos a las clases variable o invariable en español es relativamente predecible a partir de sus terminaciones, de manera que se obtiene un sistema moderadamente estable ${ }^{6}$.

${ }^{6}$ En relación con los adjetivos variables de (6c) y (6d), que conforman el grupo tercero en la clasificación de la RAE (1973), en esta obra se subraya «que la mayor parte de los adjetivos que componen este grupo tercero tienen significación de persona y que muchos de ellos funcionan al mismo tiempo como sustantivos, dotados de la capacidad de formar sustantivos con -a» (p. 194), una propiedad sobre la que volveremos a incidir en la sección 3.2. 
La correspondencia entre terminaciones y clases formales es más intricada en los adjetivos del catalán, tal como se ilustra en (7).

(7) Implicaciones formales del sistema de adjetivos del catalán

a. Implicación 1: las formas con masculino en -o son variables:

$$
\text { fof-o fof-a }
$$

b. Implicación 2: las formas con masculino en $-u$ son variables:

ate-u ate-a

c. Implicación 3: las formas con masculino en -a son invariables:

belg-a

agrícol-a

d. Implicación 4: las formas con masculino acabado en consonante son variables:

Pero:

$\begin{array}{ll}\text { clar- } \varnothing & \text { clar-a } \\ \text { fort- } \varnothing & \text { fort-a } \\ \text { cortés- } \varnothing & \text { cortes-a } \\ \text { valent- } \varnothing & \text { valent-a } \\ \text { tranquil- } \varnothing & \text { tranquil-1-a }\end{array}$

$\begin{array}{ll}\text { escolar- } \varnothing & \text { elemental- } \varnothing \\ \text { fàcil- } \varnothing & \text { potent- } \varnothing \\ \text { delirant- } \varnothing & \text { servil- } \varnothing \\ \text { feliç- } \varnothing & \\ \text { (cf. feliç-os 'felices, } & \\ \end{array}$

e. Implicación 5: las formas acabadas en -e son invariables:

$\begin{array}{ll}\text { notabl-e } & \text { simpl-e } \\ \text { alegr-e } & \text { còmod-e }\end{array}$

Pero:

$$
\text { ampl-e, ampl-a negr-e, negr-a }
$$

En general, existen pocos casos en que una terminación remita a un único patrón y, además, estos casos de relación unívoca corresponden a formas poco frecuentes. Así, como en español, los adjetivos cuyo masculino acaba en -o, como fof-o, son variables (7a), y los adjetivos cuyo masculino acaba en - $a$, como belg- $a$, invariables $(7 \mathrm{c})$. En catalán, son también variables los adjetivos con la terminación - $u$ en el masculino, como ate-u (7b). En estos tres casos, la presencia de las terminaciones $-o,-a y-u$ en el masculino sirve como pista para adscribir los adjetivos a una única clase, aunque, eso sí, se trata de terminaciones poco frecuentes.

El patrón más común en la lengua es la alternancia $\varnothing \sim-a$, como en clar- $\varnothing$ - clar-a (7d). A diferencia del español, en que la terminación $\varnothing$ se asocia normalmente con adjetivos invariables, en catalán suele corresponder a adjetivos variables, si bien con un elenco de excepciones bastante numeroso. En efecto, como resultado de la eliminación histórica de las vocales átonas finales diferentes de $a$ en catalán, los adjetivos variables del patrón $\varnothing \sim-a$, procedentes en su mayoría de adjetivos de tres terminaciones en latín, son formalmente idénticos en el masculino a los adje- 
tivos invariables acabados en consonante provenientes de adjetivos de dos terminaciones en latín, como escolar. Algunos de estos adjetivos invariables acabados en consonante en catalán pertenecen a subclases que se pueden identificar a partir de alguna propiedad formal. Así, igual que en español, encontramos en este grupo adjetivos acabados en - $a \xi ̧,-i c ̧,-o c ̧$, como feliç 'feliz' (que son, sin embargo, variables en el plural); en los sufijos - aly -ar, como elemental 'elemental'; adjetivos llanos acabados en -il, como fàcil, o comparativos como millor 'mejor'. También suelen ser invariables los adjetivos acabados en -el o en -il, como fidel 'fiel' o servil 'servil', y en -ent o en -ant, como potent 'potente' o delirant 'delirante'. Como consecuencia de que algunas de estas subclases incluyen también adjetivos variables, el problema fundamental para la estabilidad del sistema es que ciertas formas invariables acabadas en consonante como potent, delirant o servil son completamente indistinguibles en el masculino singular de adjetivos variables como valent- $\varnothing$ 'valiente, MASC.' - valent- $a$ 'valiente, FEM.', comboiant- $\varnothing$ 'despierto, listo' - comboiant-a 'despierta, lista' o tranquil- $\varnothing$ 'tranquilo' - tranquil.l-a 'tranquila'. Así pues, el grupo de adjetivos invariables acabados en consonante constituye en catalán una clase claramente inestable; dada su ambigüedad formal, se podría decir lo mismo de la clase $\varnothing \sim-a$, que, sin embargo, por criterios cuantitativos (Wurzel 1987: 79), es el paradigma preferente de la lengua y se debe considerar un patrón estable.

Por último, los adjetivos cuyo masculino acaba en -e suelen ser, como en español, invariables, como notable (7e). Ahora bien, también son numerosos los ejemplos de adjetivos variables con el patrón -e - -a, como ampl-e - ampl-a. Esta convergencia formal se origina sobre todo a causa de la eliminación histórica de las vocales finales diferentes de $a$ en catalán; debido a este proceso, en algunos adjetivos variables se añade una vocal epentética $e$ para evitar la creación de grupos consonánticos complejos en posición final de palabra: por ej., *ampl o *negr. Aunque existen algunas terminaciones que típicamente son invariables, como -ble (notable) o -aire (manaire 'mandón, MASC. y FEM.'), la existencia de adjetivos invariables fuera de esos grupos como simple o alegre 'alegre', junto con formas variables de estructura equivalente como ampl-e - ampl-a o negr-e - negr-a, difumina los límites de ambas subclases. En definitiva, aunque en catalán existen ciertas generalizaciones robustas referidas a la relación entre terminaciones y clases formales, las dos terminaciones más frecuentes en el masculino singular $-\varnothing \mathrm{y}-\ell-$ no permiten adscribir los adjetivos con certeza a la clase de formas variables o invariables, respectivamente.

\section{CORRECCIÓN FORMAL DE LOS ADJETIVOS INVARIABLES DEL CATALÁN}

Como consecuencia de la falta de iconicidad y de transparencia de las formas sincréticas y de la indefinición formal de la clase de adjetivos invariables en catalán, existe en esta lengua una gran tendencia a la regularización de las formas invariables, esto es, a la eliminación de la ambigüedad formal de las terminaciones. Básicamente, encontramos los siguientes tres tipos de regularización de adjetivos invariables, ordenados de mayor a menor frecuencia: a) regularización de adjetivos de 
la terminación $\varnothing$, siguiendo el patrón de máxima iconicidad $\varnothing \sim-a$ (sección 3.1); b) regularización de adjetivos con la terminación -e, siguiendo el patrón de mínima iconicidad $-e--a, \mathrm{y} c$ ) regularización de adjetivos acabados en -a (sobre todo, con la terminación -ista), siguiendo también el patrón -e - -a (sección 3.2). En español, existen procesos de regularización equivalentes a los del catalán, pero son menos comunes, ya que, como veremos, aunque incrementan la iconicidad y la transparencia formal, no mejoran la estabilidad de las clases formales.

\subsection{EXTENSIÓN DE FORMAS ANALÓGICAS SEGÚN EL PATRÓN $\varnothing \sim-A$}

Tras la eliminación de las vocales finales latinas diferentes de - $a$ en catalán, se observa, desde los primeros tiempos de la lengua, una fuerte tendencia a crear femeninos analógicos para las formas invariables sin marca flexiva, como fort- $\varnothing_{\mathrm{o}}$ verd- $\varnothing$. Los femeninos respectivos, fort- $a$ y verd- $a$, se generan a partir de formas del patrón dominante $\varnothing \sim-a$ como curt- $\varnothing$ 'corto' - curt- $a$ 'corta', siguiendo el esquema de analogía proporcional propuesto en su día por los neogramáticos (8).

$$
\begin{aligned}
& \text { Creación analógica de femeninos a partir del patrón } \varnothing \sim-a \text { en catalán } \\
& \text { curt- } \varnothing: \text { curt- } \mathrm{a}=\text { fort- } \varnothing: \mathrm{x} \quad> \\
& \text { curt- } \varnothing: \text { curt- } \mathrm{a}=\text { fort } \varnothing \text { : fort-a }
\end{aligned}
$$

Los beneficios estructurales del proceso analógico que crea forta o verda son múltiples. De entrada, se reduce la clase de palabras excepcionalmente invariables, es decir, el sistema gana en transparencia formal. Además, el nuevo patrón analógico se integra en el modelo morfológico dominante de la lengua, el modelo $\varnothing \sim-a$, que no solo es el que contiene más casos, sino también el patrón no marcado; por lo tanto, la migración de formas invariables al patrón $\varnothing--a$ aumenta la estabilidad del sistema. Finalmente, la regularización implica un aumento máximo de la iconicidad formal, puesto que se genera una forma femenina añadiendo elementos a la forma masculina básica. Por todo ello, no sorprende que este tipo de regularizaciones sea muy frecuente, sobre todo en lenguas como el catalán, con elisión de la marca flexiva en el masculino (Lausberg 1973: 116; Casanova 1983: 244; Duarte y Alsina 1986: 24-25).

En catalán, pues, la creación de femeninos analógicos según el esquema $\varnothing \sim-a$ aumenta la transparencia y la iconicidad de los adjetivos y la coherencia global del sistema. Por este motivo, los adjetivos invariables sin flexión son proclives a regularizarse en todas las épocas de la lengua. Las nuevas formas analógicas, sin embargo, no se generan ni se difunden al mismo ritmo (Casanova 1983: 158), sino que siguen el patrón de difusión léxica característico de los cambios lingüísticos graduales. Ilustramos este punto con algunos ejemplos representativos en la tabla 1, realizada a partir del Corpus Informatitzat del Català antic (CICA). Como muestran los datos, las formas analógicas comuna ('común, FEM.', de comú 'común, MASC.') y dolça ('dulce, FEM.', de dolç 'dulce, MASC.'), que aparecen ya en la segunda mitad del siglo xiII, y la forma folla ('loca', de foll 'loco'), que se documenta por vez 
primera un poco más tarde, se consolidan pronto, mientras que otras formas como verda y forta no se encuentran hasta el final del siglo XIV y el inicio del XV, respectivamente, y tardan más en arraigar; por último, ciertos femeninos analógicos como un hipotético servila, a partir de servil, ni tan siquiera parecen haber existido7. Así pues, la aparición de las formas analógicas puede ser muy antigua o relativamente moderna o incluso no haberse producido nunca, y la pervivencia de las formas sincréticas en el tiempo no es tampoco en absoluto homogénea.

\begin{tabular}{|c|c|c|c|c|c|c|c|c|c|c|c|c|c|}
\hline \multicolumn{14}{|c|}{$\begin{array}{c}\text { TABLA 1. REGULARIZACIÓN DE ADJETIVOS INVARIABLES SEGÚN EL PATRÓN } \\
\text { MÁXIMAMENTE ICÓNICO - } \varnothing--A \text { : RECUENTO Y, ENTRE PARÉNTESIS, FRECUENCIA, } \\
\text { POR SIGLOS, DE FORMAS FEMENINAS SIN MARCA - } A \text { EXPLÍCITA (IZQUIERDA) } \\
\text { Y CON MARCA - } A \text { EXPLÍCITA (DERECHA) }\end{array}$} \\
\hline & & \multicolumn{2}{|c|}{ comú comuna } & \multirow{2}{*}{$\begin{array}{c}\text { dolç } \\
3 \\
(37,5\end{array}$} & \multirow{2}{*}{$\begin{array}{c}\text { dolça } \\
5 \\
62,5)\end{array}$} & \multirow{2}{*}{ foll } & \multirow{2}{*}{ folla } & \multirow{2}{*}{$\begin{array}{c}\text { verd } \\
12 \\
(\mathbf{1 0 0}\end{array}$} & \multirow{2}{*}{$\begin{array}{c}\text { verda } \\
0 \\
-)\end{array}$} & \multirow{2}{*}{$\begin{array}{c}\text { fort } \\
9 \\
(100\end{array}$} & \multirow{2}{*}{$\begin{array}{c}\text { forta } \\
0 \\
-)\end{array}$} & \multicolumn{2}{|c|}{ servil servila } \\
\hline XIII & $\begin{array}{c}\text { Casos } \\
(\%)\end{array}$ & $\begin{array}{c}1 \\
(4,8\end{array}$ & $\begin{array}{c}20 \\
95,2)\end{array}$ & & & & & & & & & $\begin{array}{c}2 \\
(100 \\
\end{array}$ & $\begin{array}{c}0 \\
-)\end{array}$ \\
\hline XIV & $\begin{array}{c}\text { Casos } \\
(\%)\end{array}$ & $\begin{array}{l}0 \\
(-\end{array}$ & $\begin{array}{c}176 \\
\mathbf{1 0 0})\end{array}$ & $\begin{array}{l}0 \\
(-\end{array}$ & & $\begin{array}{l}0 \\
(-\end{array}$ & $\begin{array}{c}67 \\
100)\end{array}$ & $\begin{array}{c}19 \\
(70,4\end{array}$ & $\begin{array}{c}8 \\
29,6\end{array}$ & $\begin{array}{c}189 \\
(\mathbf{1 0 0}\end{array}$ & $\begin{array}{c}0 \\
-)\end{array}$ & $\begin{array}{c}1 \\
(\mathbf{1 0 0}\end{array}$ & $\begin{array}{c}0 \\
-)\end{array}$ \\
\hline $\mathrm{XV}$ & $\begin{array}{l}\text { Casos } \\
(\%)\end{array}$ & $\begin{array}{l}0 \\
(- \\
\end{array}$ & $\begin{array}{c}105 \\
\mathbf{1 0 0})\end{array}$ & $\begin{array}{l}0 \\
(- \\
\end{array}$ & $\begin{array}{r}15 \\
10 \\
\end{array}$ & $\begin{array}{c}1 \\
(1,1\end{array}$ & $\begin{array}{c}87 \\
98,9) \\
\end{array}$ & $\begin{array}{c}23 \\
(65,7 \\
\end{array}$ & $\begin{array}{c}12 \\
34,3) \\
\end{array}$ & $\begin{array}{c}309 \\
(99,7\end{array}$ & $\begin{array}{c}1 \\
0,3)\end{array}$ & $\begin{array}{c}5 \\
(\mathbf{1 0 0}\end{array}$ & $\begin{array}{c}0 \\
-)\end{array}$ \\
\hline $\mathrm{XVI}$ & $\begin{array}{c}\text { Casos } \\
(\%)\end{array}$ & $\begin{array}{l}0 \\
(-\end{array}$ & $\begin{array}{c}46 \\
100)\end{array}$ & $\begin{array}{l}0 \\
(-\end{array}$ & $\begin{array}{c}22 \\
100)\end{array}$ & $\begin{array}{l}0 \\
(-\end{array}$ & $\begin{array}{c}11 \\
100)\end{array}$ & $\begin{array}{c}2 \\
(11,8\end{array}$ & $\begin{array}{c}15 \\
88,2)\end{array}$ & $\begin{array}{c}37 \\
(\mathbf{9 2 , 5}\end{array}$ & $\begin{array}{c}3 \\
7,5)\end{array}$ & $\begin{array}{c}1 \\
(100\end{array}$ & $\begin{array}{c}0 \\
-)\end{array}$ \\
\hline
\end{tabular}

Además de cambios como el formalizado en (8), el catalán presenta un tipo minoritario de regularización, específico de los adjetivos invariables con marca $\varnothing$ en el singular que acaban ortográficamente en la consonante sibilante -ç. Así, como hemos indicado en (7d), el adjetivo feliç, invariable en el singular, cuenta con una forma para el masculino plural, feliç-os, y otra forma diferente para el femenino plural, felic-es. En este caso, la antigua forma del plural era felices para los dos géneros y se creó un masculino específico con la vocal -o-, que, como hemos visto en (7), se asocia unívocamente con el masculino (para una discusión del origen de estas formas, v. Moll 1952: \$251; Coromines 1974: 204-207, 311-313; Recasens 2017: 63-64). La diferenciación de las formas del plural en este patrón supone un aumento de la transparencia formal y una mejora mínima de la iconicidad construccional, porque el masculino, distinto del femenino, no se forma añadiendo un segmento a la forma masculina sino cambiando una vocal. Tipológicamente, sin embargo, esta

7 Tampoco aparecen en el CICA femeninos analógicos para amargant 'amargo', dòcil 'dócil', fácil o feliç. En el lenguaje popular se encuentran casos de regularización analógica de estos adjetivos según el patrón $\varnothing--a$ no aceptados por la normativa, como amarganta, dòcila, fàcila o feliça (Badia 1951: \$117, III, 6), todos documentados en el más actual Corpus Textual Informatitzat de la Llengua Catalana $(C T I L C)$. El rastro de estas formas se puede reseguir también en textos recientes de internet; por ejemplo, la forma feliça se puede encontrar en el título de un artículo de Julià Guillamon: «Nois, això és l'arcàdia feliça!» ‘CChicos, esto es la arcadia feliz!' (La Vanguardia, 11/01/2012). 
regularización es extraña, ya que va en contra del principio de aceptación del sincretismo, según el cual, en elementos marcados del paradigma como el plural, se tolera mejor la homonimia que en los no marcados ( $c f$. Wheeler 1993: 108; entre los antecedentes de este principio de naturalidad el mismo Wheeler cita el universal 37 de Greenberg 1963: 58: «A language never has more gender categories in nonsingular numbers than in the singular»). Normalmente, pues, esperaríamos una mayor diferenciación en el singular que en el plural, que es la parte marcada del paradigma, como ocurre en adjetivos del tipo - $e--a$ como ample, con diferenciación en el singular: ample - ampla, pero una única forma para el plural: amples.

En español, por otra parte, aunque los femeninos analógicos a partir de formas acabadas en $\varnothing$ también existen, son mucho menos frecuentes que en catalán: se limitan, como hemos indicado, a adjetivos acabados en -or, como traidor; en -ón, como bretón; en -és, como francés, y a un grupo de gentilicios como andaluz (v. Menéndez Pidal 1987: 219-220; Urrutia y Álvarez 2001: 76-77). Desde el punto de vista de la transparencia y de la iconicidad, esto es, sin considerar el sistema en su conjunto, formas analógicas del tipo traidor- $\varnothing$ - traidor-a son tan idóneas como en catalán. En cambio, respecto de la coherencia del sistema, empeoran las relaciones implicatorias entre terminaciones y clases formales, ya que las formas acabadas en consonante son típicamente invariables en español; además, las formas resultantes se encuadran en el patrón $\varnothing--a$ y no en el modelo dominante de la lengua: $-o \sim-a$. En definitiva, aunque localmente se mejore la naturalidad en los adjetivos regularizados, la generación de formas analógicas del tipo $\varnothing \sim-a$ reduce la estabilidad global del sistema, por lo que la tendencia a seguir este modelo no es tan fuerte como en catalán. La menor tendencia del español a crear este tipo de formas analógicas confirma la idea, expuesta en Mayerthaler (1987) y Wurzel (1987), de que los principios de naturalidad dependientes del sistema, como la necesidad de mantener la estabilidad formal de las clases, prevalecen sobre los principios independientes del sistema, como la tendencia al aumento de la transparencia y de la iconicidad de las formas. Con todo, la creación efectiva de formas analógicas que empeoran la definición de las clases y, en consecuencia, el sistema globalmente, parece contradecir la visión -estricta- de estos autores de que la satisfacción de los principios independientes del sistema está supeditada a la no transgresión de los principios dependientes del sistema (v., sobre este punto, la discusión de Wheeler 1993). Este debate volverá a surgir en la siguiente sección, donde apuntamos a una posible explicación compatible con la propuesta de Mayerthaler (1987) y Wurzel (1987).

\subsection{EXTENSIÓN DE FORMAS ANALÓGICAS SEGÚN EL PATRÓN $-E \sim-A$}

A diferencia del español, el catalán cuenta con un buen número de adjetivos variables en género según el patrón $-e--a$ que provienen de formas con variación de género ya en latín, como ample - ampla o negre - negra. Como hemos indicado en la sección 2.2, con la elisión de las vocales finales diferentes de - $a$ en el paso al catalán, estos adjetivos perdieron la vocal final original para añadir una vocal epentética [e] con el objeto de evitar grupos consonánticos finales complejos. En 
términos de naturalidad, como hemos señalado en la sección 2.1, el modelo -e - $a$ es transparente y mínimamente icónico. Desde el punto de vista de la estabilidad global del sistema, sin embargo, estas formas variables complican la gramática, puesto que la terminación -e, que funcionaba como un indicio de la clase invariable (Casanova 1985: 131), pasa a convertirse en formalmente ambigua, lo que obliga a los hablantes a realizar un esfuerzo adicional de aprendizaje. Además, las formas con la alternancia $-e--a$ generan un patrón secundario respecto del principal de la lengua: $\varnothing \sim-a$. La menor idoneidad formal del modelo $-e--a$ es la responsable del poco éxito a lo largo de la historia del catalán de regularizaciones de adjetivos invariables acabados en -e, como alegre, según el esquema de analogía proporcional que resumimos en (9). Los mismos argumentos se pueden aplicar a la regularización de las formas invariables acabadas en $-a$ según el patrón $-e--a$, que en la práctica se reduce a las formas nominales -adjetivos y sustantivos- con el sufijo-ista, como evangelista 'evangelista' (10).

(9) Creación analógica de femeninos a partir del patrón -e - - $a$ en catalán negr-e $:$ negr-a $=$ alegr-e $:$ x negr-e : negr-a = alegr-e $:$ alegr-a

Creación analógica de masculinos a partir del patrón - $e--a$ en catalán negr-a $:$ negr-e $=$ evangelist-a $: \mathrm{x}>$ negr-a $:$ negr-e $=$ evangelist-a $:$ evangelist-e

En la tabla 2 presentamos una muestra de la documentación del $C I C A$ referida a adjetivos acabados en -e que inicialmente eran invariables, como alegre, noble 'noble' o notable, o que eran variables en origen pero son actualmente invariables, como doble y simple. Los adjetivos acabados en -ble tienden en general a mantenerse como invariables, como ilustra notable. La fuerza de este grupo ha atraído a este patrón a formas inicialmente variables, como el adjetivo doble, acabado también en -ble, o el adjetivo simple, asociado a doble porque aparece en contextos similares. También ha propiciado que el adjetivo noble, que en la lengua antigua presentaba bastantes ocurrencias de femenino acabadas en - $a$, haya vuelto al modelo invariable original. En cambio, la palabra alegre, mayoritariamente variable en la documentación del CICA, no muestra aún en esta época una tendencia clara a adaptarse al patrón invariable, aunque más adelante pasaría a ser invariable. En ninguno de los casos las formas variables, que mejoran la transparencia y la iconicidad, han llegado a desplazar a las formas sincréticas, que son las propuestas en la normativa actual (AVL 2006; IEC 2016), seguramente por la asociación entre la terminación -e y la clase invariable de adjetivos (Casanova 1985: 131). 


\begin{tabular}{|c|c|c|c|c|c|c|c|c|c|c|c|}
\hline \multicolumn{12}{|c|}{$\begin{array}{l}\text { TABLA 2. VARIACIÓN EN EL FEMENINO DE LOS ADJETIVOS (ACTUALMENTE) } \\
\text { INVARIABLES ACABADOS EN - E ADAPTADOS SEGÚN EL PATRÓN MÍNIMAMENTE } \\
\text { ICÓNICO - }--A \text { : RECUENTO Y, ENTRE PARÉNTESIS, FRECUENCIA, POR SIGLOS, } \\
\text { DE FORMAS FEMENINAS CON MARCA - } E \text { (IZQUIERDA) Y CON MARCA - } A \text { (DERECHA) }\end{array}$} \\
\hline & & alegre & alegra & noble & nobla & notable & notabla & doble & dobla & simple & simpla \\
\hline S. XIII & $\begin{array}{c}\text { Casos } \\
(\%)\end{array}$ & $\begin{array}{c}4 \\
(80 \\
\end{array}$ & $\begin{array}{c}1 \\
20) \\
\end{array}$ & $\begin{array}{c}6 \\
(13,3 \\
\end{array}$ & $\begin{array}{c}39 \\
86,7) \\
\end{array}$ & - & & $\begin{array}{r}2 \\
(5 \\
\end{array}$ & $\begin{array}{c}38 \\
95)\end{array}$ & $\begin{array}{c}16 \\
(18,8\end{array}$ & $\begin{array}{c}69 \\
81,2)\end{array}$ \\
\hline S. XIV & $\begin{array}{c}\text { Casos } \\
(\%)\end{array}$ & $\begin{array}{c}5 \\
(14,3\end{array}$ & $\begin{array}{c}30 \\
85,7)\end{array}$ & $\begin{array}{c}141 \\
(72,7\end{array}$ & $\begin{array}{c}53 \\
27,3)\end{array}$ & $\begin{array}{c}12 \\
(92,3\end{array}$ & $\begin{array}{c}1 \\
7,7)\end{array}$ & $\begin{array}{c}4 \\
(50\end{array}$ & $\begin{array}{c}4 \\
50)\end{array}$ & $\begin{array}{c}28 \\
(54,9\end{array}$ & $\begin{array}{c}23 \\
45,1)\end{array}$ \\
\hline S. XV & $\begin{array}{c}\text { Casos } \\
(\%)\end{array}$ & $\begin{array}{r}15 \\
(9,3\end{array}$ & $\begin{array}{c}147 \\
90,7)\end{array}$ & $\begin{array}{c}211 \\
(80,2\end{array}$ & $\begin{array}{c}52 \\
19,8)\end{array}$ & $\begin{array}{c}83 \\
(78,3\end{array}$ & $\begin{array}{c}23 \\
21,7)\end{array}$ & $\begin{array}{c}5 \\
(35,7\end{array}$ & $\begin{array}{c}9 \\
64,3)\end{array}$ & $\begin{array}{c}18 \\
(58,1\end{array}$ & $\begin{array}{c}13 \\
41,9)\end{array}$ \\
\hline S. XVI & $\begin{array}{c}\text { Casos } \\
(\%)\end{array}$ & $\begin{array}{c}10 \\
(28,6\end{array}$ & $\begin{array}{c}25 \\
71,4)\end{array}$ & $\begin{array}{c}71 \\
(98,6\end{array}$ & $\begin{array}{c}1 \\
1,4)\end{array}$ & $\begin{array}{c}20 \\
(\mathbf{1 0 0}\end{array}$ & $\begin{array}{c}0 \\
-)\end{array}$ & $\begin{array}{c}3 \\
(75\end{array}$ & $\begin{array}{c}1 \\
25)\end{array}$ & $\begin{array}{c}21 \\
(95,5\end{array}$ & $\begin{array}{c}1 \\
4,5)\end{array}$ \\
\hline
\end{tabular}

En el caso de los masculinos analógicos del tipo evangeliste, comienzan a aparecer en la documentación a partir del siglo XIV, y su uso va creciendo de manera que en siglo Xvi la terminación -iste es la forma predominante y pasa a ser la única forma a partir del siglo XVII (Casanova 1984: 261-262; AVL 2006: 96). Con todo, la normativa actual recomienda la forma invariable para la escritura en el ámbito general (IEC 2016: 163), si bien se acepta la diferencia de género en el ámbito local valenciano (AVL 2006: 96) y en los usos orales de la lengua (IEC 2016: 163). Ilustramos este punto en la tabla 3 con las variantes para los sustantivos evangelista y psalmista 'salmista'. Como se puede observar, las regularizaciones según este patrón no son muy frecuentes, aunque las formas masculinas acabadas en $-e$ parecen ir aumentando de frecuencia con el tiempo.

\begin{tabular}{|c|c|c|c|c|c|}
\hline \multicolumn{6}{|c|}{$\begin{array}{l}\text { TABLA 3. VARIACIÓN EN EL MASCULINO DE LOS ELEMENTOS NOMINALES INVARIA- } \\
\text { BLES ACABADOS EN - A ADAPTADOS SEGÚN EL PATRÓN MÍNIMAMENTE ICÓNICO } \\
-E--A \text { : RECUENTO Y, ENTRE PARÉNTESIS, FRECUENCIA, POR SIGLOS, DE FORMAS } \\
\text { MASCULINAS CON MARCA - } A \text { (IZQUIERDA) Y CON MARCA - E (DERECHA) }\end{array}$} \\
\hline & & evangelista & evangeliste & psalmista & psalmiste \\
\hline S. XIII & $\begin{array}{c}\text { Casos } \\
(\%)\end{array}$ & $\begin{array}{c}15 \\
(93,8\end{array}$ & $\begin{array}{c}1 \\
6,2)\end{array}$ & - & \\
\hline S. XIV & $\begin{array}{c}\text { Casos } \\
(\%)\end{array}$ & $\begin{array}{c}9 \\
(60\end{array}$ & $\begin{array}{c}6 \\
40)\end{array}$ & $\begin{array}{c}3 \\
(100 \\
\end{array}$ & $\begin{array}{c}0 \\
-) \\
\end{array}$ \\
\hline S. XV & $\begin{array}{c}\text { Casos } \\
(\%)\end{array}$ & $\begin{array}{c}122 \\
(92,4\end{array}$ & $\begin{array}{c}10 \\
7,6)\end{array}$ & $\begin{array}{c}23 \\
(79,3\end{array}$ & $\begin{array}{c}6 \\
20,7)\end{array}$ \\
\hline S. XVI & $\begin{array}{c}\text { Casos } \\
(\%)\end{array}$ & $\begin{array}{c}5 \\
(35,7\end{array}$ & $\begin{array}{c}9 \\
64,3)\end{array}$ & $\begin{array}{c}3 \\
(75\end{array}$ & $\begin{array}{c}1 \\
25)\end{array}$ \\
\hline
\end{tabular}

En conjunto, pues, observamos que las regularizaciones que siguen el patrón $-e--a$ son poco frecuentes en catalán y no siempre han tenido éxito. En español estas regularizaciones son también muy poco comunes: además de liante, -a, que hemos presentado en la sección 2.2, conforman el grupo de formas acabadas en 
$-e--a$ creadas por analogía, según datos extraídos del $D L E$, los adjetivos atorrante, $-a$; negociante, $-a$; tunante, $-a$, $\mathrm{y}$, con limitaciones, pariente, $-a^{8}$; en el lenguaje popular se documentan otras formas como dominante, $-a$, o mangante, $-a^{9}$. La poca frecuencia del cambio se explica porque no existe en esta lengua una red de relaciones, una clase formal, que empuje a los adjetivos en esa dirección. En cambio, sí que existen motivos para evitar la aparición de un patrón variable $-e \sim-a$, puesto que complicaría la gramática al eliminar del sistema la asociación entre la terminación -e y la invariabilidad del género. En este sentido, conviene subrayar que, cuando las formas que contienen la terminación -ista se regularizan, se inscriben en el patrón no marcado - $o \sim-a$, como ocurre con el sustantivo modisto.

En definitiva, comprobamos que, cuando el aumento en la iconicidad no es máximo y la estabilidad de las clases del sistema o no mejora o empeora directamente, las dos lenguas analizadas muestran una tendencia menor a la creación de formas transparentes para el género, es decir, de femeninos y, en algún caso, de masculinos analógicos. Esto demuestra que los principios dependientes del sistema prevalecen sobre las tendencias independientes del sistema, como la iconicidad y la transparencia. Ahora bien, la existencia de algunas formas analógicas como lianta en español y evangeliste en catalán invita a considerar que los últimos principios se pueden imponer ocasionalmente a los principios dependientes del sistema, como la estabilidad de las clases, en contra de lo propuesto por Mayerthaler (1987) y Wurzel (1987). Con todo, en nuestra opinión, la situación es bastante más compleja, con redes de relaciones que van más allá de los adjetivos y de sus clases formales. Así, ejemplos como lianta o evangeliste se pueden explicar porque los adjetivos no son un sistema estanco en la lengua, sino que están fuertemente influidos por la forma de

${ }^{8}$ Estos pocos casos de alternancia $-e \sim-a$ en adjetivos contrastan con la mayor frecuencia de sustantivos de persona en -e dotados de moción genérica (RAE 1973: 192), entre los cuales 16 con la terminación -ante, $-a$, como gigant-e, $-a$ (además de algunos femeninos en -anta con entrada propia como almiranta, comandanta o gobernanta), y 16 más con la terminación -ente, -a, como sirvient-e, $-a$ (además de dos que solo tienen femenino: sergenta y parturienta). Esta mayor tendencia de los sustantivos a crear femeninos analógicos se puede ilustrar con la frase La cabeza de la giganta es gigante, en que solo el sustantivo admite la marca de género femenino. Por eso, la posibilidad de que un adjetivo pueda usarse también como un sustantivo, como ocurre con gigant-e, - $a$, parece favorecer la creación de femeninos analógicos (en esta misma línea, el femenino serventa 'sirvienta' es de creación temprana en catalán y la forma servent, sin moción de género, no se utiliza ni una sola vez en los textos del CICA para referirse al femenino; $v$. $g$. Jiménez y Lloret, en prensa). Además, son más frecuentes las regularizaciones en adjetivos que se pueden usar para caracterizar personas; así, mientras que liante ha desarrollado un femenino analógico, no existe un femenino paralelo para el adjetivo picante (excepto cuando se usa como sustantivo, por ej., en apodos).

9 Estas formas analógicas del español se pueden rastrear fácilmente en las redes sociales, en algunos casos combinadas con sustantivos de la misma terminación aceptados en el $D L E$, como infanta: "Que impute a la Infanta 'Manganta' y juzgue a la 'pareja feliz' (https://www.change.org/p/ juez-jos\%C3\%A9-castro-arag\%C3\%B3n-que-impute-a-la-infanta-manganta-y-juzgue-a-la-parejafeliz), o mezclando formas variables, como dominanta, con formas invariables de origen similar, como impertinente: «Qué impertinente y qué dominanta es la madre de MJ» (https://twitter.com/i/ web/status/1100507780648239105). 
los sustantivos. Estos últimos tienen mayor tendencia a regularizarse, seguramente por la mayor relevancia del rasgo de género en esta clase ${ }^{10}$. En este sentido, el carácter doble, como formadoras de adjetivos y también de sustantivos, de terminaciones como -ante, -ente, en español, o-ista, -ent, -ant, en catalán, seguramente ha debido favorecer la extensión analógica de las formas femeninas originalmente creadas para los sustantivos a los adjetivos, siguiendo el esquema analógico que ilustramos en (11). La restricción de femeninos como sirvienta o giganta a su uso como sustantivos en el $D L E$, así como de las formas equivalentes del catalán serventa y geganta en el $D I E C$, o el hecho de que las primeras muestras de la forma -iste en la documentación catalana correspondan a sustantivos (evangeliste, canoniste 'canonista, MASC.', juriste 'jurista, MASC.', legiste 'legista, MASC.', sofiste 'sofista, MASC.', sobre todo a partir del siglo XIV; Casanova 1984: 261), reforzaría esa suposición. Esta asociación entre tipos diferentes de palabras se ha producido a pesar de generar clases morfológicas menores y menos estables en el caso de los adjetivos: en español, por ej., se sustituyen elementos de la clase invariable en - $e$, mayoritaria, por elementos transparentes y mínimamente icónicos con el modelo $e--a$, que oscurecen la relación entre la terminación - e y la clase invariable.

$$
\begin{aligned}
& \text { Creación analógica de masculinos a partir del patrón }-e \sim-a \text { en español } \\
& \text { infant-e }: \text { infant-a }=\text { mangant-e }: \mathrm{x}> \\
& \text { infant-e }: \text { infant-a }=\text { mangant-e }: \text { mangant-a }
\end{aligned}
$$

\section{CONCLUSIÓN}

En este artículo hemos mostrado que el catalán se caracteriza por una fuerte tendencia a la conversión de las formas invariables en cuanto al género en variables -en transparentes-, si bien esta preferencia no se manifiesta con la misma intensidad en todas las clases de adjetivos como consecuencia de la interacción entre diferentes principios de naturalidad. Por un lado, es más fuerte en las clases de adjetivos en que la regularización permite obtener un patrón de iconicidad máxima, como en fort- $\varnothing \sim$ fort- $a$, mientras que es menos intensa cuando la iconicidad de las formas resultantes es mínima, como en los pares alegr-e - alegr-a o evangelist-e - evangelist-a. Por otro, la intensidad de la tendencia está condicionada por la frecuencia del patrón resultante, de manera que, de nuevo, las regularizaciones del tipo fort- $\varnothing$ fort-a, según el patrón $\varnothing \sim-a$ predominante en la lengua, resultan favorecidas por encima de las regularizaciones según el patrón $-e--a$.

La situación del español es diferente, sobre todo porque los cambios analógicos según el modelo traidor- $\varnothing \sim$ traidor-a, que generan formas de máxima iconicidad y son muy naturales si se centra la atención localmente en las formas resultan-

10 Este factor es subrayado explícitamente por Badia (1951: \$117.III) cuando apunta que las formas analógicas se han ido aceptando especialmente en los casos de sustantivación. 
tes, aumentan la frecuencia de una clase variable minoritaria en la lengua, el patrón $\varnothing \sim-a$, y, por lo tanto, empeoran la estabilidad global del sistema. Este pequeño matiz es suficiente para entender por qué el patrón $\varnothing \sim-a$ no tiene en español tanta fuerza de atracción como en catalán. Por otra parte, la sólida asociación entre la terminación -e y la clase invariable de adjetivos o, desde otro punto de vista, la escasez de adjetivos variables que se inscriban en el modelo $-e--a$, así como la mínima iconicidad de las formas de este patrón, justifican que el cambio analógico en adjetivos del tipo liante, $-a$ sea virtualmente inexistente en espańol, paralelamente a lo que sucede en catalán.

En cuanto a la difusión de las nuevas formas, conviene destacar que la tendencia a la regularización se observa en todas las épocas del catalán y del español, con un patrón de difusión típico de los cambios que afectan al léxico, sin que se haya llegado a un punto de equilibrio definitivo. Por eso, el proceso, todavía activo, sigue generando en las dos lenguas nuevas formas analógicas que van muchas veces por delante de la prescripción normativa, como ocurre con femeninos como amarganta, feliça o fácila, en catalán, o como dominanta y manganta, en español.

En definitiva, los datos muestran que la probabilidad de aplicar procesos que mejoren la naturalidad de una forma concreta -en el caso estudiado, que aumenten su transparencia y su iconicidad formal- depende, como apuntan los trabajos de la morfología natural, de la organización del sistema en su conjunto y, en concreto, de la generación o del reforzamiento de clases formales de adjetivos estables, que se puedan definir claramente a partir de propiedades extramorfológicas (concretamente, fonológicas en el caso de las marcas de género del catalán y del español). 


\section{BIBLIOGRAFÍA}

Acadèmia Valenciana de la Llengua (AVL) (2006): Gramàtica normativa valenciana, València: Publicacions de l’Acadèmia Valenciana de la Llengua. URL: http://www.avl.gva.es.

Badia i Margarit, Antoni M. (1951): Gramática histórica catalana, Madrid: Gredos. [Edición catalana: Gramàtica històrica catalana, València: Tres i Quatre, 1981].

Casanova, Emili (1983): «Sobre la moció de gènere dels adjectius de la 3a declinació llatina en català», Boletín de la Sociedad Castellonense de Cultura 59: 243-264.

Casanova, Emili (1984): "Sobre la moció del sufix iste / ista», Boletín de la Sociedad Castellonense de Cultura 60: 259-267.

Casanova, Emili (1985): «Sobre la moció de gènere en català medieval: El cas dels sufixos adjectivals cultes -ABILIS, IBILIS», en Actes du XVIIème Congrès International de Linguistique et Philologie Romanes (Aix-en-Provence, 29 août-3 septembre 1983), vol. 3, Aix-en-Provence: Université de Provence, 121-134.

CICA = Torruella, Joan (dir.) (junto con Manuel Pérez Saldanya y Josep Martines) (2006): Corpus Informatitzat del Català Antic. URL: http://seneca.uab.es/sfi/CICA; 25/05/2019.

Clua, Esteve (2002): "Gènere i nombre en els noms i en els adjectius», en Joan Solà, Maria-Rosa Lloret, Joan Mascaró y Manuel Pérez Saldanya (dirs.), Gramàtica del català contemporani, vol. 1, Barcelona: Empúries, 483-534.

Coromines, Joan (1974): Lleures i converses d'un filòleg, 2. a ed., Barcelona: Club Editor.

CTILC = Institut d'Estudis Catalans (2005): Corpus Textual Informatitzat de la Llengua Catalana. URL: https://ctlc.iec.cat/; 25/05/2019.

DECat = Coromines, Joan (1980-1991): Diccionari etimologic $i$ complementari de la llengua catalana, 9 vol., Barcelona: Curial.

DIEC = Institut d'Estudis Catalans (2007): Diccionari de la llengua catalana, Barcelona: Edicions 62 / Enciclopèdia Catalana. URL: https://dlc.iec.cat/; 25/05/2019.

DLE = Real Academia Española (2014): Diccionario de la lengua española (23. ${ }^{a}$ ed.), Madrid: Espasa. URL: http://www.rae.es/rae.html; 25/05/2019.

Duarte i Montserrat, Carles y Àlex Alsina i Keith (1986): Gramàtica històrica del català, vol. 2, Barcelona: Curial.

Dressler, Wofgang U. (1985): «On the predictiveness of Natural Morphology», Journal of Linguistics 21: 321-337.

Dressler, Wofgang U. (1987): «Introduction», en Wolfgang U. Dressler, Willi Mayerthaler y Oswald Panagl (eds.), Leitmotifs in Natural Morphology, Amsterdam, Philadelphia: John Benjamins, 3-22,

Fabra, Pompeu (1956): Gramàtica catalana, Barcelona: Teide.

Greenberg, Joseph H. (1963): «Some universals of grammar with particular reference to the order of meaningful elements», en Joseph H. Greenberg (ed.), Universals of Language, Cambridge, Mass.: MIT Press, 73-113.

Institut d'Estudis Catalans (IEC) (2016): Gramàtica de la llengua catalana, Barcelona: Institut d'Estudis Catalans. 
Jiménez, Jesús y Maria-Rosa LloRet (en prensa): «La morfologia flexiva: el gènere i les terminacions nominals», en Josep Martines y Manuel Pérez Saldanya (eds.), Gramàtica del català antic, Amsterdam, Philadelphia: John Benjamins.

LAUSBERG, Heinrich (1973): Lingüistica románica, vol. II, Madrid: Gredos.

Lloret, Maria-Rosa (2013): «Diferències en les terminacions flexives dels elements nominals», en Esteve Clua y Maria-Rosa Lloret (eds.), Qüestions de morfologia flexiva i lèxica del català. Volum d'homenatge a Joaquim Viaplana, Alacant: Institut Interuniversitari de Filologia Valenciana, 245-278.

Mascaró, Joan (1985): Morfologia, Barcelona: Enciclopèdia Catalana.

Mayerthaler, Willi (1987): «I. System-independent morphological naturalness», en Wolfgang U. Dressler, Willi Mayerthaler y Oswald Panagl (eds.), Leitmotifs in Natural Morphology, Amsterdam, Philadelphia: John Benjamins, 25-58.

Mayerthaler, Willi (1988): Morphological Naturalness, Ann Arbor: Karoma Press. [Edición original alemana: Morphologische Natürlichkeit, Wiesbaden: Athenaion, 1981].

Menéndez Pidal, Ramón (1987): Manual de gramática histórica, 19. ${ }^{a}$ ed., Madrid: Espasa-Calpe.

Moll, Francesc de Borja (1952): Gramática histórica catalana, Madrid: Gredos. [Edición catalana: Gramàtica històrica catalana, València: Publicacions de la Universitat de València, 2006].

Pérez Saldanya, Manuel (1998): Del llatí al català. Morfosintaxi verbal històrica, València: Universitat de València.

Pérez Saldanya, Manuel, Manuel Sifre y Júlia Todolí (2004): Morfologia catalana, Barcelona: Universitat Oberta de Catalunya.

Real Academia Española (RAE) (1973): Esbozo de una Nueva Gramática de la Lengua Española, Madrid: Espasa-Calpe.

Real Academia Española (RAE) y Asociación de Academias de la lengua Española (ASALE) (2009): Nueva gramática de la lengua española. Morfología. Sintaxis I, Madrid: Espasa.

Recasens i Vives, Daniel (2017): Fonètica històrica del català, Barcelona: Institut d'Estudis Catalans.

Urrutia, Hernán y Manuela Álvarez (2001): Esquema de morfosintaxis histórica del español, 3. ${ }^{\circledR}$ ed., Bilbao: Universidad de Deusto.

Vennemann, Theo (1978): «Phonetic analogy and conceptual analogy», en Philip Baldi y Ronald N. Werth (eds.), Readings in Historical Phonology: Chapters in the Theory of Sound Change, University Park, Penn.: Pennsylvania State University Press, 258-274.

WheELER, Max W. (1993). «On the hierarchy of naturalness principles in inflectional morphology», Journal of Linguistics 29: 55-111.

WurzeL, Wolfgang Ullrich (1987): «II. System-dependent morphological naturalness in inflection», en Wolfgang U. Dressler, Willi Mayerthaler y Oswald Panagl (eds.), Leitmotifs in Natural Morphology, Amsterdam, Philadelphia: John Benjamins, 59-96.

WurzeL, Wolfgang Ullrich (1989): Inflectional Morphology and Naturalness, Dordrecht: Kluwer Academic Publishers. [Edición original alemana: Flexionsmorphologie und Natürlichkeit: Ein Beitrag zur morphologischen Theoriebildung, Berlín: Akademie-Verlag, 1984]. 